\title{
ON THE b-STABLE SET POLYTOPE OF GRAPHS WITHOUT BAD $K_{4}{ }^{*}$
}

\author{
DION GIJSWIJT ${ }^{\dagger}$ AND ALEXANDER SCHRIJVER $\ddagger$
}

\begin{abstract}
We prove that for a graph $G=(V, E)$ without bad $K_{4}$ subdivision, and for $b \in \mathbf{Z}_{+}^{V \cup E}$, the $b$-stable set polytope is determined by the system of constraints determined by the vertices, edges, and odd circuits. We also prove that this system is totally dual integral. This relates to t-perfect graphs.
\end{abstract}

Key words. t-perfect, graph, polytope, stable set

AMS subject classifications. 05C69, 90C27, 90C57

DOI. $10.1137 /$ S0895480102417343

Let $G=(V, E)$ be a graph and let $b \in \mathbf{Z}_{+}^{V \cup E}$. Then a $b$-stable set in $G$ is a vector $x \in \mathbf{Z}_{+}^{V}$ satisfying $x_{v} \leq b_{v}$ for every vertex $v$ and $x_{u}+x_{v} \leq b_{u v}$ for every edge $u v$. The $b$-stable set polytope of $G$ is defined as the convex hull of the $b$-stable sets in $G$.

We will use the following notation. For sets $B \subseteq A$ and a vector $x \in \mathbf{R}^{A}$, let $\chi^{B}$ be the characteristic vector of $B$ and let $x(B):=x^{T} \chi^{B}$. For an edge $\{u, v\}$ we will use the shorthand notation $u v$.

The vectors in the $b$-stable set polytope obviously satisfy the following system of inequalities:

(i) $0 \leq x_{v} \leq b_{v} \quad$ for each $v \in V$;

(ii) $x_{u}+x_{v} \leq b_{u v}$ for each edge $u v \in E$;

(iii) $x(V C) \leq\left\lfloor\frac{1}{2} b(E C)\right\rfloor$ for each odd circuit $C$.

We call a graph $G$ t-perfect with respect to $b$ if the $b$-stable set polytope is determined by (1). Since each integral vector satisfying (1) is a $b$-stable set, the polytope determined by (1) equals the $b$-stable set polytope if and only if it is integral. We call a graph $G$ strongly t-perfect with respect to $b$ if system $(1)$ is totally dual integral.

For any weight function $w \in \mathbf{Z}_{+}^{V}$ and any $b \in \mathbf{Z}_{+}^{V \cup E}$, denote by $\alpha(G, b, w)$ the maximum $w$-weight $w^{T} x$ of a $b$-stable set $x$ in $G$. Define a $w$-cover as a family of vertices, edges, and odd circuits in $G$ that covers each vertex $v$ at least $w_{v}$ times. The $b$-cost of a $w$-cover is defined as the sum of the costs of its elements, where the cost of a vertex $v$ equals $b_{v}$, the cost of an edge $e$ equals $b_{e}$, and the cost of an odd circuit $C$ equals $\left\lfloor\frac{1}{2} b(E C)\right\rfloor$. Denote by $\tilde{\rho}(G, b, w)$ the minimum cost of a $w$-cover. Strong t-perfection can now be characterized equivalently as follows: a graph $G=(V, E)$ is strongly t-perfect with respect to $b$ if and only if $\alpha(G, b, w)=\tilde{\rho}(G, b, w)$ for every weight function $w \in \mathbf{Z}_{+}^{V}$.

Call a subdivision of $K_{4}$ odd if each triangle of $K_{4}$ has become an odd circuit. An odd subdivision of $K_{4}$ is called bad if there are no two disjoint edges $e, f$ of $K_{4}$ such

\footnotetext{
* Received by the editors November 8, 2002; accepted for publication (in revised form) March 21, 2003; published electronically June 25, 2003.

http://www.siam.org/journals/sidma/16-3/41734.html

†Department of Mathematics, University of Amsterdam, Plantage Muidergracht 24, $1018 \mathrm{TV}$ Amsterdam, The Netherlands (gijswijt@science.uva.nl).

$\ddagger$ CWI, Kruislaan 413, 1098 SJ Amsterdam, The Netherlands, and Department of Mathematics, University of Amsterdam, Plantage Muidergracht 24, 1018 TV Amsterdam, The Netherlands (lex cwi.nl).
} 
that $e$ and $f$ are not subdivided and the other four edges have become even length paths. We say that a graph has a bad $K_{4}$ subdivision if it has a subgraph that is a bad $K_{4}$ subdivision.

In [4], it was proved that a graph has no bad $K_{4}$ subdivision if and only if each subgraph is t-perfect with respect to the all-one vector. Here the "if" part follows from the fact that a bad $K_{4}$ subdivision is not t-perfect with respect to the all-one vector (see [1]). In [5], it was proved that graphs without bad $K_{4}$ subdivision are also strongly t-perfect with respect to the all-one vector. In this paper we prove that graphs having no bad $K_{4}$ subdivision are strongly t-perfect with respect to every $b \in \mathbf{Z}_{+}^{V \cup E}$, which implies our theorem.

THEOREM. Let $G=(V, E)$ be a graph. Then the following are equivalent:

(i) $G$ has no bad $K_{4}$ subdivision.

(ii) $G$ is t-perfect with respect to each $b \in \mathbf{Z}_{+}^{V \cup E}$.

(iii) $G$ is strongly t-perfect with respect to each $b \in \mathbf{Z}_{+}^{V \cup E}$.

Proof. If $G$ satisfies (ii), then also each subgraph of $G$ satisfies (ii). So the implication (ii) $\Longrightarrow$ (i) follows from the fact that a bad $K_{4}$ subdivision is not t-perfect with respect to the all-one vector (see [1]).

The implication (iii) $\Longrightarrow$ (ii) follows from the fact that any totally dual integral system with integral right-hand side determines an integral polyhedron.

To prove the implication (i) $\Longrightarrow$ (iii), it will be convenient to first prove the implication (i) $\Longrightarrow$ (ii). Let $G=(V, E)$ be a graph without bad $K_{4}$ subdivision, and let $b \in \mathbf{Z}_{+}^{V \cup E}$. We show that the polytope $P$ determined by (1) is integral. Suppose that $x$ is a nonintegral vertex of $P$. Let $x^{\prime}$ be defined by $x_{v}^{\prime}:=x_{v}-\left\lfloor x_{v}\right\rfloor$ for every vertex $v$, and let $b^{\prime}$ be defined by $b_{v}^{\prime}:=b_{v}-\left\lfloor x_{v}\right\rfloor$ for every vertex $v$ and $b_{e}^{\prime}:=b_{e}-\left\lfloor x_{u}\right\rfloor-\left\lfloor x_{v}\right\rfloor$ for every edge $e=u v$. Then $x^{\prime}$ is a nonintegral vertex of the polytope determined by (1) with $b$ replaced by $b^{\prime}$. Let $G^{\prime}:=(V, F)$, where $F:=\left\{e \in E \mid b_{e}^{\prime}=1\right\}$. Since $G^{\prime}$ has no bad $K_{4}$ subdivision and $x^{\prime}$ satisfies the constraints (1) for the graph $G^{\prime}$ and the all-one vector $\chi^{V \cup F}$ instead of $b, x^{\prime}$ is a convex combination of incidence vectors of stable sets in $G^{\prime}$ by [5]. Each of these incidence vectors is a $b^{\prime}$-stable set. Hence $x^{\prime}$ is a convex combination of $b^{\prime}$-stable sets in $G$, a contradiction. This proves the implication (i) $\Longrightarrow$ (ii).

The remainder of this proof consists of showing the implication (i) $\Longrightarrow$ (iii). The idea is to reduce the general statement to the case in which $b$ is the all-one vector.

Suppose the implication (i) $\Longrightarrow$ (iii) is false. Let the graph $G=(V, E)$ and $b \in \mathbf{Z}_{+}^{V \cup E}$ form a counter example with (first) $|V|+|E|$ minimal and (second) $b(V)$ minimal. Let $w \in \mathbf{Z}_{+}^{V}$ be any weight function for which $\alpha(G, b, w)<\tilde{\rho}(G, b, w)$. Note that by the minimality of $G$, we know that $G$ has no isolated vertices. We observe the following facts about $w$ and $b$.

Claim 1.

(i) $\alpha(G, b, w)<\alpha\left(G-e,\left.b\right|_{G-e}, w\right)$ for each edge $e \in E$,

(ii) $b_{u v}<b_{u}+b_{v}$ for each edge $u v \in E$,

(iii) $1 \leq b_{u} \leq b_{u v}$ for each edge $u v \in E$,

(iv) $1 \leq w_{v}$ for each vertex $v \in V$.

Proof. By the minimality of $G$, we know that

$$
\alpha(G, b, w)<\tilde{\rho}(G, b, w) \leq \tilde{\rho}\left(G-e,\left.b\right|_{G-e}, w\right)=\alpha\left(G-e,\left.b\right|_{G-e}, w\right) .
$$

This gives (i). If for some edge $u v$ we have $b_{u v} \geq b_{u}+b_{v}$, then every $\left.b\right|_{G-u v}$-stable set in $G-u v$ is a $b$-stable set in $G$, contradicting (i). Hence we have (ii). Suppose 
that $b_{u}>b_{u v}$ for some edge $u v$. Let $b^{\prime}:=b-\chi^{u}$. Now we have

$$
\alpha\left(G, b^{\prime}, w\right)=\alpha(G, b, w)<\tilde{\rho}(G, b, w)=\tilde{\rho}\left(G, b^{\prime}, w\right)
$$

contradicting the minimality of $b$. Hence we have $0 \leq b_{u v}-b_{v}<b_{u} \leq b_{u v}$, and (iii) follows. Suppose that $w_{v}=0$ for some vertex $v$. Let $b^{\prime}:=\left.b\right|_{G-v}$ and $w^{\prime}:=\left.w\right|_{G-v}$. Then

$$
\alpha\left(G-v, b^{\prime}, w^{\prime}\right)=\alpha(G, b, w)<\tilde{\rho}(G, b, w) \leq \tilde{\rho}\left(G-v, b^{\prime}, w^{\prime}\right),
$$

contradicting the minimality of $G$. Hence we have (iv).

For the $b$-stable sets of maximum weight we have the following.

Claim 2. Let $x$ be a b-stable set of $w$-weight $w^{T} x=\alpha(G, b, w)$. Then $x_{v} \leq 1$ for each $v \in V$.

Proof. To see this, suppose that $x_{v}>1$ for some vertex $v$. Let $x^{\prime}:=x-\chi^{v}$ and $b^{\prime}:=b-\chi^{\{v\} \cup \delta(v)}$. For any $b^{\prime}$-stable set $\tilde{x}$ in $G$, we have

$$
w^{T} \tilde{x}=w^{T}\left(\tilde{x}+\chi^{v}\right)-w_{v} \leq \alpha(G, b, w)-w_{v}=w^{T} x^{\prime},
$$

and hence $x^{\prime}$ is a maximum $w$-weight $b^{\prime}$-stable set in $G$. By minimality of $b$, there exists a $w$-cover $F$ of $b^{\prime}$-cost $\tilde{\rho}\left(G, b^{\prime}, w\right)=\alpha\left(G, b^{\prime}, w\right)$.

Since $x_{v}>1$, we have $x_{v}^{\prime}>0$, and hence by "complementary slackness" $v$ is covered exactly $w_{v}$ times by $F$. This implies that $F$ has $b$-cost

$$
\tilde{\rho}\left(G, b^{\prime}, w\right)+w_{v}=\alpha\left(G, b^{\prime}, w\right)+w_{v}=\alpha(G, b, w),
$$

a contradiction.

Claim 3. For every edge $f \in E$ we have $b_{f} \leq 2$.

Proof. Suppose that Claim 3 is not true and that we have $b_{f} \geq 3$ for some edge $f=u v$. Let $w^{\prime}:=w+N \cdot \chi^{f}$, where $N:=w(V)+1$. Then

$$
\alpha\left(G, b, w^{\prime}\right)=\tilde{\rho}\left(G, b, w^{\prime}\right),
$$

since otherwise by Claim 2 applied to $w^{\prime}$ we have for any maximum $w^{\prime}$-weight $b$-stable set $x$ the inequality

$$
{w^{\prime}}^{T} x=w^{T} x+N\left(x_{u}+x_{v}\right) \leq N-1+2 N<3 N,
$$

while $x^{\prime}:=b_{u} \chi^{u}+\left(b_{f}-b_{u}\right) \chi^{v}$ is a $b$-stable set of $w^{\prime}$-weight

$$
w^{\prime T} x^{\prime} \geq N \cdot b_{f} \geq 3 N
$$

contradicting the optimality of $x$.

So we can choose $w$ such that

$$
\begin{aligned}
\alpha(G, b, w) & <\tilde{\rho}(G, b, w), \\
\alpha\left(G, b, w+\chi^{f}\right) & =\tilde{\rho}\left(G, b, w+\chi^{f}\right) .
\end{aligned}
$$

Let $F:=\left\{v_{1}, \ldots, v_{r}, e_{1}, \ldots, e_{s}, C_{1}, \ldots, C_{t}\right\}$ be a minimum $b$-cost $w+\chi^{f}$-cover, where the $v_{i}$ are vertices, the $e_{i}$ are edges, and the $C_{i}$ are odd circuits. Note that none of the $e_{i}$ is the edge $f$, since otherwise $\tilde{\rho}(G, b, w) \leq \tilde{\rho}\left(G, b, w+\chi^{f}\right)-b_{f}$, which would imply that $\tilde{\rho}(G, b, w) \leq \alpha\left(G, b, w+\chi^{f}\right)-b_{f} \leq \alpha(G, b, w)$. Let $G^{\prime}:=G-f$, let 
$b^{\prime}:=\left.b\right|_{G^{\prime}}$, and let $x^{\prime}$ be a maximum $w$-weight $b^{\prime}$-stable set in $G^{\prime}$. Then $\alpha\left(G^{\prime}, b^{\prime}, w\right) \geq$ $\alpha(G, b, w)+1$ by Claim 1 , and hence

$$
x^{\prime}(f)>b_{f} .
$$

For any odd circuit $C$ traversing $f$, we have

$$
x^{\prime}(V C) \leq\left\lfloor\frac{1}{2} b(E C)\right\rfloor+\frac{1}{2}\left(x^{\prime}(f)-b_{f}+1\right),
$$

since $2 x^{\prime}(V C) \leq x^{\prime}(f)+b(E C-f)=x^{\prime}(f)+b(E C)-b_{f}$. Now let $l$ be the number of circuits in $F$ traversing $f$. We obtain

$$
\begin{aligned}
\tilde{\rho}\left(G, b, w+\chi^{f}\right) & =\alpha\left(G, b, w+\chi^{f}\right) \leq \alpha(G, b, w)+b_{f} \leq \alpha\left(G^{\prime}, b^{\prime}, w\right)-1+b_{f} \\
& =w^{T} x^{\prime}-1+b_{f}=\left(w+\chi^{f}\right)^{T} x^{\prime}-\left(x^{\prime}(f)-b_{f}+1\right) \\
& \leq-\left(x^{\prime}(f)-b_{f}+1\right)+\sum_{i=1}^{r} x^{\prime}\left(v_{i}\right)+\sum_{i=1}^{s} x^{\prime}\left(e_{i}\right)+\sum_{i=1}^{t} x^{\prime}\left(V C_{i}\right) \\
& \leq\left(\frac{1}{2} l-1\right)\left(x^{\prime}(f)-b_{f}+1\right)+\sum_{i=1}^{r} b_{v_{i}}+\sum_{i=1}^{s} b_{e_{i}}+\sum_{i=1}^{t}\left\lfloor\frac{1}{2} b\left(E C_{i}\right)\right\rfloor \\
& =\left(\frac{1}{2} l-1\right)\left(x^{\prime}(f)-b_{f}+1\right)+\tilde{\rho}\left(G, b, w+\chi^{f}\right) .
\end{aligned}
$$

Hence we have $(l-2)\left(x^{\prime}(f)-b_{f}+1\right) \geq 0$. Since $x^{\prime}(f)-b_{f}+1>0$ by (4), we have $l \geq 2$.

We may assume that $C_{1}$ and $C_{2}$ traverse $f$. Decompose the cycle $E C_{1} \Delta E C_{2}$ into circuits $C_{1}^{\prime}, \ldots, C_{q}^{\prime}$, where $C_{1}^{\prime}, \ldots, C_{p}^{\prime}$ are odd and $C_{p+1}^{\prime}, \ldots, C_{q}^{\prime}$ are even. Choose in each $C_{i}^{\prime}$ with $i=p+1, \ldots, q$ a perfect matching $M_{i}$ with $b\left(M_{i}\right) \leq \frac{1}{2} b\left(E C_{i}^{\prime}\right)$. Now the circuits $C_{1}$ and $C_{2}$ are removed from the cover $F$, and the circuits $C_{1}^{\prime}, \ldots, C_{p}^{\prime}$, the edges in the matchings $M_{p+1}, \ldots, M_{q}$, and the edges in $E C_{1} \cap E C_{2}$ are added to the cover. This gives a $w+\chi^{f}$-cover $F^{\prime}$ of $b$-cost

(7) $\tilde{\rho}\left(G, b, w+\chi^{f}\right)-\left\lfloor\frac{1}{2} b\left(E C_{1}\right)\right\rfloor-\left\lfloor\frac{1}{2} b\left(E C_{2}\right)\right\rfloor$

$$
+b\left(E C_{1} \cap E C_{2}\right)+\sum_{i=1}^{p}\left\lfloor\frac{1}{2} b\left(E C_{i}^{\prime}\right)\right\rfloor+\sum_{i=p+1}^{q} b\left(M_{i}\right)
$$

$$
\leq \tilde{\rho}\left(G, b, w+\chi^{f}\right)-\frac{1}{2}\left(b\left(E C_{1}\right)+b\left(E C_{2}\right)-2\right)+\frac{1}{2}\left(b\left(E C_{1} \Delta E C_{2}\right)\right)+b\left(E C_{1} \cap E C_{2}\right)
$$

$$
=\tilde{\rho}\left(G, b, w+\chi^{f}\right)+1 \text {. }
$$

Hence $F^{\prime}-f$ is a $w$-cover of $b$-cost at most $\tilde{\rho}\left(G, b, w+\chi^{f}\right)+1-b_{f}$. This implies that

(8) $\alpha(G, b, w) \leq \tilde{\rho}(G, b, w)-1 \leq \tilde{\rho}\left(G, b, w+\chi^{f}\right)-b_{f}$

$$
=\alpha\left(G, b, w+\chi^{f}\right)-b_{f} \leq \alpha(G, b, w) .
$$

So we have equality throughout and, in particular, we obtain

$$
\alpha\left(G, b, w+\chi^{f}\right)=\alpha(G, b, w)+b_{f} .
$$

Let $x$ be a maximum $w+\chi^{f}$-weight $b$-stable set in $G$. Then

$$
\alpha(G, b, w)+b_{f}=\left(w+\chi^{f}\right)^{T} x=w^{T} x+x(f) \leq \alpha(G, b, w)+b_{f},
$$


and hence $x(f)=b_{f}$ and $x$ is a maximum $w$-weight $b$-stable set. However, $x(f)=$ $b_{f} \geq 3$ implies that $x_{u}>1$ or $x_{v}>1$, contradicting Claim 2.

Partition the vertex set $V$ into $V_{1}:=\left\{v \in V \mid b_{v}=1\right\}$ and $V_{2}:=\left\{v \in V \mid b_{v}=2\right\}$. Thus by Claim 1 , we know that the edges $e$ spanned by $V_{1}$ have $b_{e}=1$ and the other edges have $b_{e}=2$. We now prove the following claim.

Claim 4. Either $V_{1}=\emptyset$ or $V_{2}=\emptyset$.

Proof. To prove the claim, take $w$ with $\alpha(G, b, w)<\tilde{\rho}(G, b, w)$ such that $w(V)$ is minimal. We first prove the following:

$$
\text { If } b_{v}=1 \text { for some vertex } v
$$

then there exists a maximum $w$-weight $b$-stable set $x$ with $x_{v}=0$.

Indeed, let $w^{\prime}:=w-\chi^{v}$. By the minimality of $w$, we have

$$
\alpha\left(G, b, w^{\prime}\right)+1=\tilde{\rho}\left(G, b, w^{\prime}\right)+1 \geq \tilde{\rho}(G, b, w) \geq \alpha(G, b, w)+1 .
$$

Hence $\alpha\left(G, b, w^{\prime}\right)=\alpha(G, b, w)$, implying that there exists a maximum $w$-weight $b$ stable set $x$ satisfying $x_{v}=0$.

Similarly, we have the following:

If $b_{e}=1$ for some edge $e$,

then there exists a maximum $w$-weight $b$-stable set $x$ with $x(e)=0$.

To see this, let $w^{\prime}:=w-\chi^{e}$. By the minimality of $w$, we have

$$
\alpha\left(G, b, w^{\prime}\right)+1=\tilde{\rho}\left(G, b, w^{\prime}\right)+1 \geq \tilde{\rho}(G, b, w) \geq \alpha(G, b, w)+1 .
$$

Hence $\alpha\left(G, b, w^{\prime}\right)=\alpha(G, b, w)$, implying that there exists a maximum $w$-weight $b$ stable set $x$ satisfying $x(e)=0$.

Consider an edge $e=u v$ with $u \in V_{1}$ and $v \in V_{2}$. By (9), there is a maximum $w$-weight $b$-stable set $x$ with $x_{u}=0$. By Claim 2, we know that $x_{v} \leq 1$. Hence $x(e) \leq 1<2=b_{e}$. So we have that

for each edge $e \in \delta\left(V_{1}\right)$,

there is a maximum $w$-weight $b$-stable set $x$ with $x(e)<b_{e}$.

Next consider an odd circuit traversing an edge in $\delta\left(V_{1}\right)$. We have that

for each odd circuit $C$ traversing an edge in $\delta\left(V_{1}\right)$, there is a maximum $w$-weight $b$-stable set $x$ with $x(V C)<\left\lfloor\frac{1}{2} b(E C)\right\rfloor$.

Indeed, let $C$ be an odd circuit traversing an edge in $\delta\left(V_{1}\right)$ and suppose that $C$ does not traverse an edge spanned by $V_{1}$. Let $u \in V_{1}$ be a vertex traversed by $C$. By (9), there is a maximum $w$-weight $b$-stable set $x$ with $x_{u}=0$. By Claim 2 we have $x(V C) \leq|V C|-1<|V C|=\left\lfloor\frac{1}{2} b(E C)\right\rfloor$.

Thus we may assume that $C$ traverses an edge spanned by $U_{1}$. Then $(:$ hats three consecutive vertices $t, u$, and $v$ with $t, u \in V_{1}$, and $v \in V_{2}$. By (10) there is a maximum $u$-weight $b$-stable set $x$ with $x(t u)=0=b_{t u}-1$. By Claim 2 we hav $x_{v} \leq 1$, and hence $x(u v) \leq 1 \leq b_{u v}-1$. Thus $2 x(V C) \leq b(E C)-2$, and hence $x(V C)<\left\lfloor\frac{1}{2} b(E C)\right\rfloor$.

Now suppose that $V_{1}$ and $V_{2}$ are nonempty. By minimality of $G$, we know that there is at least one edge $e \in \delta\left(V_{1}\right)$. Let $G^{\prime}:=G-e$, let $b^{\prime}:=\left.b\right|_{i^{\prime}}$, and let $x^{\prime}$ be 
a maximum $w$-weight $b^{\prime}$-stable set in $G^{\prime}$. Let $x$ maximize $w^{T} x$ over the $b$-stable set polytope of $G$ such that $x$ is in general position on the face of optimal solutions. Then by (11) and (12), $x(e)<b_{e}$ and $x(V C)<\left\lfloor\frac{1}{2} b(E C)\right\rfloor$ for each odd circuit traversing $e$. Hence there is a $0<\lambda \leq 1$ such that $\tilde{x}:=(1-\lambda) x+\lambda x^{\prime}$ satisfies the system of constraints (1). By the implication (i) $\Longrightarrow$ (ii), $\tilde{x}$ belongs to the $b$-stable set polytope of $G$. However, $w^{T} \tilde{x}>w^{T} x$, since $w^{T} x^{\prime}=\alpha\left(G^{\prime}, b^{\prime}, w\right)>\alpha(G, b, w)=w^{T} x$ by Claim 1. This contradicts the optimality of $x$. So either $V_{1}$ or $V_{2}$ is empty.

If $b$ is the all-one vector, the total dual integrality of (1) follows from [5]. So $V_{1}$ is empty, and hence $b_{e}=2$ for every edge $e$ and $b_{v}=2$ for every vertex $v$. Denote by a $2 w$-edge cover a vector $y \in \mathbf{Z}_{+}^{E}$ with $y(\delta(v)) \geq 2 w_{v}$ for every vertex $v \in V$. It is easy to see that for any $2 w$-edge cover $y$ and any 2-stable set $x$, we have $w^{T} x \leq \sum_{e \in E} y_{e} \frac{1}{2} \sum_{v \in e} x(v) \leq y(E)$. By a theorem of Gallai (see [2]), $G$ has a 2-stable set $x$ and a $2 w$-edge cover such that $w^{T} x=y(E)$. Denote by $U_{y}$ the set of vertices $v$ for which $y(\delta(v))$ is odd. Let $x$ be a 2-stable set and let $y$ be a $2 w$-edge cover such that $w^{T} x=y(E)$ and $\left|U_{y}\right|$ is minimal.

If $U_{y} \neq \emptyset$, then there is a simple path $P$ connecting two vertices in $U_{y}$ with $y_{e} \geq 1$ for each $e \in E P$. Let $M$ be a maximum size matching in $P$. Then $y^{\prime}:=y+\chi^{E P}-2 \chi^{M}$ is a $2 w$-edge cover with $y^{\prime}(E) \leq y(E)$ and $\left|U_{y^{\prime}}\right|=\left|U_{y}\right|-2$, a contradiction. So $y(\delta(v))$ is even for every vertex $v$ and we can write $y=\chi^{E C_{1}}+\cdots+\chi^{E C_{r}}+\chi^{E C_{1}^{\prime}}+\cdots+\chi^{E C_{s}^{\prime}}$ for odd circuits $C_{1}, \ldots, C_{r}$ and even circuits $C_{1}^{\prime}, \ldots, C_{s}^{\prime}$. Let $M_{i}$ be a perfect matching in $C_{i}^{\prime}$ for $i=1, \ldots, s$. Then $C_{1}, \ldots, C_{r}$ together with the edges in the matchings $M_{1}, \ldots, M_{s}$ give a $w$-cover of $b$-cost $y(E)=w^{T} x$. Since $x$ is a $b$-stable set, this implies that $\tilde{\rho}(G, b, w) \leq \alpha(G, b, w)$, contradicting the choice of $w$. This concludes the proof of the theorem.

Remark. Let $G=(V, E)$ be a graph with $E \times V$ incidence matrix $M$. In [3] it was proved that the matrix

$$
\left(\begin{array}{c}
I \\
-I \\
M \\
-M
\end{array}\right)
$$

has Chvátal rank at most 1 if and only if $G$ has no odd $K_{4}$ subdivision. The equivalence of (i) and (ii) of the theorem above has the following reformulation in terms of the Chvátal rank: the matrix

$$
\left(\begin{array}{c}
I \\
-I \\
M
\end{array}\right)
$$

has Chvátal rank at most 1 if and only if $G$ has no bad $K_{4}$ subdivision.

\section{REFERENCES}

[1] F. Barahona And A.R. Mahjoub, Composition of graphs and polyhedra III: Graphs with no $W_{4}$ minor, SIAM J. Discrete Math., 7 (1994), pp. 372-389.

[2] T. GallaI, Maximum-minimum Sätze über Graphen, Acta Math. Acad. Sci. Hungar., 9 (1958), pp. 395-434.

[3] A.M.H. Gerards And A. Schrijver, Matrices with the Edmonds-Johnson property, Combinatorica, 6 (1986), pp. 365-379.

[4] A.M.H. Gerards AND F.B. Shepherd, The graphs with all subgraphs t-perfect, SIAM J. Discrete Math., 11 (1998), pp. 524-545.

[5] A. SchriJver, Strong t-perfection of bad-K 4 -free graphs, SIAM J. Discrete Math., 15 (2002), pp. $403-415$. 